\title{
Chirality and Spin: A Different Perspective on Enantioselective Interactions
}

\author{
Ron Naamanª, Yossi Paltiel ${ }^{b}$, and David H. Waldeck
}

\begin{abstract}
This review describes a new perspective on the role that electron spin plays in the intermolecular forces between two chiral molecules and between chiral molecules and surfaces. This different role of the spin arises from the chiral induced spin selectivity (CISS) effect which is manifest when electrons are moving in chiral molecules. Namely, it has been shown that as chiral molecules are charge polarized, the electron displacement is accompanied by spin polarization. The spin direction associated with each electric pole depends on the specific handedness of the molecule. Thus, the consideration of the dispersive forces between two molecules, or between a molecule and a substrate, must include the spin polarization which adds an enantioselective electronic term to the interaction potential. We review recent experiments that show the relation between charge polarization and spin polarization in chiral molecules. The spin polarization also affects the direction of the ferromagnetic substrate magnetic moment of a surface, upon which the chiral molecules are adsorbed.
\end{abstract}

Keywords: Enantiospecific $\cdot$ Ferromagnet $\cdot$ Hall device $\cdot$ Oligopeptide $\cdot$ Spin polarization

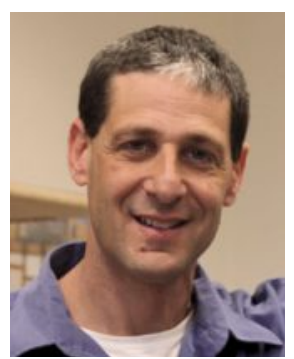

Professor Yossi Paltiel is now in the Applied Physics Department at the Hebrew University of Jerusalem Israel. Prof. Paltiel has worked for both leading high-tech industry groups and in the academic world. Since July 2009, he has led the Quantum Nano Engineering group at the Hebrew University, Israel. Paltiel is working to establish a way to incorporate quantum mechanics into room temperature 'classical' computation and reading schemes. This will provide quantum properties at nanometer scale distances, while maintaining the physical characteristics of currently

${ }^{*}$ Correspondence: Prof. R. Naaman ${ }^{a}$

E-mail: ron.naaman@weizmann.ac.il

aDepartment of Chemical and Biological Physics

Weizmann Institute of Science, Rehovot Israel 76100

${ }^{b}$ Applied Physics Department, Hebrew University

Jerusalem, Israel

'Department of Chemistry, University of Pittsburgh,

Pittsburgh PA 15260 available computer input-output devices. Professor Paltiel has published more than 100 papers in leading journals and he has 13 patents. Paltiel has a startup company named Valentis Nanotech, founded in 2013. The company utilizes nanocellulose unique properties to produce a biodegradable transparent sheet with additional controlled optical and gas/water barrier properties.

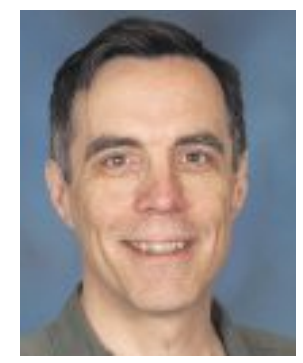

Professor David H. Waldeck is in the Chemistry Department at the University of Pittsburgh. Prof. Waldeck obtained his $\mathrm{PhD}$ in 1983 from the University of Chicago and was a postdoctoral fellow at the University of California, Berkeley before joining the Chemistry Department at Pittsburgh in 1985. Waldeck's research group investigates the nature of charge transfer and charge transport in nanostructured materials and supramolecular assemblies. An important current theme of his research program focuses on the role played by chirality and spin in charge transport and relaxation/recombination processes. Professor Waldeck has published more than 200 papers in leading journals and has been issued 2 patents.

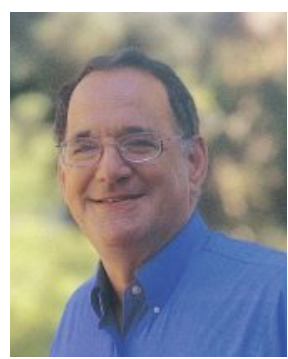

Professor Ron Naaman is in the Department of Chemical and Biological Physics at the Weizmann Institute, Israel. He obtained his $\mathrm{PhD}$ in 1978 from the Weizmann Institute and was a postdoctoral fellow at Stanford University and then at Harvard. Since 1981 he has been a faculty member at the Weizmann Institute. He has studied reactions of van der Waals molecules in molecular beams, the structure of floppy molecular systems using the Coulomb Explosion Imaging method, sensors based on hybrid molecule-semiconductor devices, and the interaction of electrons with self-assembled monolayers of biomolecules. Specifically, Naaman's group now concentrates on studying the chiral induced spin selectivity (CISS) effect and its applications. Professor Naaman has published about 280 papers and he has 16 patents.

\section{Introduction}

In $1999^{[1]}$ the first paper was published describing a new phenomenon in which electron transmission through chiral molecules is spin-dependent. This phenomenon, termed the Chiral Induced Spin Selectivity (CISS) effect, can result in relatively large 
spin polarizations, even when the molecules are only about $2 \mathrm{~nm}$ long. ${ }^{[2,3]}$ The CISS effect connects two fundamental properties of matter, chirality and electron spin, in a new way. The importance of chirality in biology and chemistry has been known since the mid- $19^{\text {th }}$ century. ${ }^{[4]}$ The existence of electron spin was not realized until the mid-1920s and was postulated, based on the spectroscopy of the hydrogen atom, by Goudsmit and Uhlenbeck. ${ }^{[5]}$ Electron spin is embedded into quantum mechanics through the Dirac equation, and plays a profound role in the structure of matter via the Pauli Exclusion Principle and symmetry constraints on the wavefunction. For example, two 'spin-less' electrons cannot occupy the same volume of space (share the same spatial wavefunction), but this becomes possible if they have opposite spins. This symmetry constraint is essential for understanding the electronic structure of the periodic table of the elements and by consequence the structure of molecules, solids, and other forms of matter.

The CISS effect and the Pauli Exclusion Principle can be combined to introduce a qualitatively new perspective on intermolecular forces that is enantiospecific, and this new quality has important implications for enantiorecognition. It is common to assume that chiral recognition and enantiomeric selectivity are related through the three-dimensional geometry of the interacting molecules, with the recognition process typically described by a 'lock and key' type model.[6] Yet, the first principles theoretical modeling of noncovalent interactions between chiral molecules fails to account for the observations quantitatively. ${ }^{[7,8]}$ This failure suggests that some essential feature(s) is not included in our current description of these interactions. ${ }^{[9,10]} \mathrm{A}$ better understanding of enantiospecific forces could impact the development of improved computational methods for predicting, more precisely, binding energies in biorecognition processes and enabling the development of novel enantioseparation methods as was shown recently in ref. [11].

We have shown that the charge reorganization in chiral molecules is accompanied by a polarization of the electron spins associated with the displaced charge. ${ }^{[12]}$ The symmetry constraints imposed by the spin polarization introduce a spin-dependent term in the interaction potential. This term gives rise to a different energy for homochiral (same enantiomers) versus heterochiral (opposite enantiomers) interactions. Calculations indicate that this contribution to the interaction energy for two molecules of the same handedness can be comparable to the average available thermal energy, $k T \cdot{ }^{[12]}$ It is worth noting that despite the fact that this effect gives rise to enantiospecific forces, they have not yet been included in modern molecular dynamics force fields.

Below we provide a short description of the physical principles underlying the CISS effect and follow it by a description of the experimental work that demonstrates the relation between charge polarization and spin polarization in chiral molecules and the effect of the spin polarization in chiral molecules on the magnetic dipole of a ferromagnetic substrate.

\section{A Physical Rationale for Enantioselectivity Based on CISS}

\subsection{The CISS Effect}

As an electron transfers between a donor and an acceptor unit through a molecular bridge, it experiences forces from its electrostatic interaction with the other electrons and the nuclei. For the case of chiral molecules, electron(s) experience a centripetal force (responsible for keeping the electrons within the molecule) that acts perpendicular to the average electron momentum; see Fig. 1. In the rest frame of the moving electrons, this force is analogous to a magnetic field $\vec{B}_{e f f}$ acting along the molecule to produce a Lorentz force on the moving electrons.

In this simplified picture, the effective magnetic field is given by $\vec{B}_{e f f}=\frac{\vec{v}}{c^{2}} \times \vec{E}_{\text {helix }}$ where $\vec{v}$ is the electron's velocity, $c$ is the speed of light, and $\vec{E}_{\text {helix }}$ is the electric field acting on the electron as a result of the potential. The spin-orbit coupling (SOC) energy is $\vec{B}_{\text {eff }} \cdot \vec{\mu}$ where $\vec{\mu}$ is the magnetic dipole of the electron. This SOC generates a Zeeman splitting between the spin states

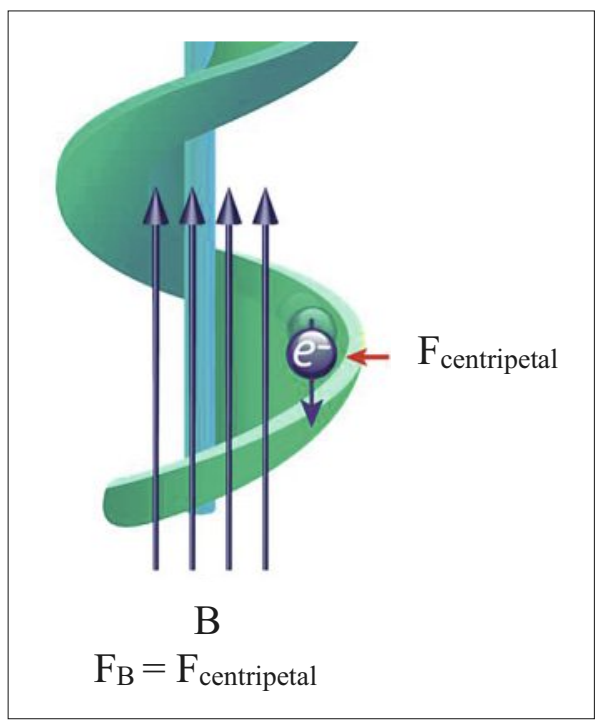

Fig. 1. When an electron is transferred along a chiral electrostatic potential, a centripetal force acts on it, perpendicular to its velocity $\vec{v}$ In the rest frame of the electron this force is like a Lorentz force that results from an 'effective magnetic' field, $\vec{B}_{e f f}$, acting along the molecule. of the transferring electron; while one spin is stabilized the other is destabilized. This results in a difference in the effective barrier heights for the electron tunneling through the molecular bridge. Because the electrons' transmission probability through the barrier depends exponentially on the barrier height, chiral molecular bridges (molecules) can have significantly different transmission probabilities for the electron's two spin components; and chiral molecules can act as spin filters. Which spin is preferred depends on the specific handedness of the molecule and on the sign of the electron velocity. ${ }^{[13]}$

\subsection{Spin Polarization and Enantio- selectivity}

The spin filtering effect, described above, implies that a dynamic charge polarization in a chiral molecule will be accompanied by spin polarization of the excess charge at the electric pole; see Fig. 2. The specific spin orientation at each pole will depend on the chirality of the molecule and the direction of the charge displacement, i.e. the electron velocity.

When a chiral molecule interacts with a substrate or two molecules interact with each other, the spin polarization imposes a symmetry constraint that affects the electron cloud overlap (Pauli exclusion). While it is known ${ }^{[14,15]}$ that electron exchange and charge penetration are important aspects of intermolecular forces at short range, currently they are not rigorously incorporated into the generation of force fields that are commonly used for molecular dynamics and biomolecular simulations. The indistinguishability of electrons leads to an exchange interaction that can stabilize the energy of two electrons with opposite spins relative to those with the same spin. ${ }^{[16,17]}$ Because spin polarization accompanies charge polarization in chiral molecules, the spin should not be neglected for describing the intermolecular forces of two closed-

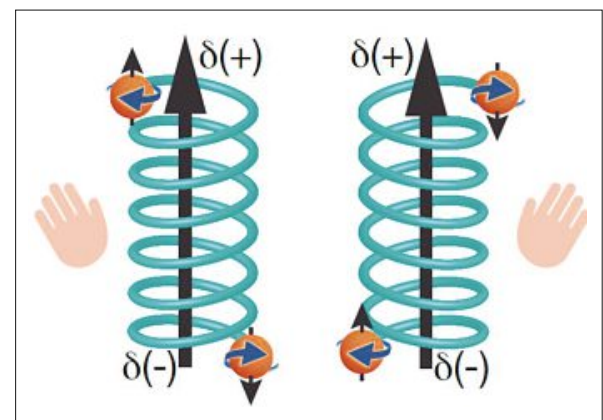

Fig. 2. A scheme describing the charge and spin polarization in two enantiomers of a chiral molecule when an electric field (black arrow) is applied. The red balls indicate the electrons with their spins (small vertical black arrows) and the angular momentum of the spin is presented by the blue horizontal arrows. 


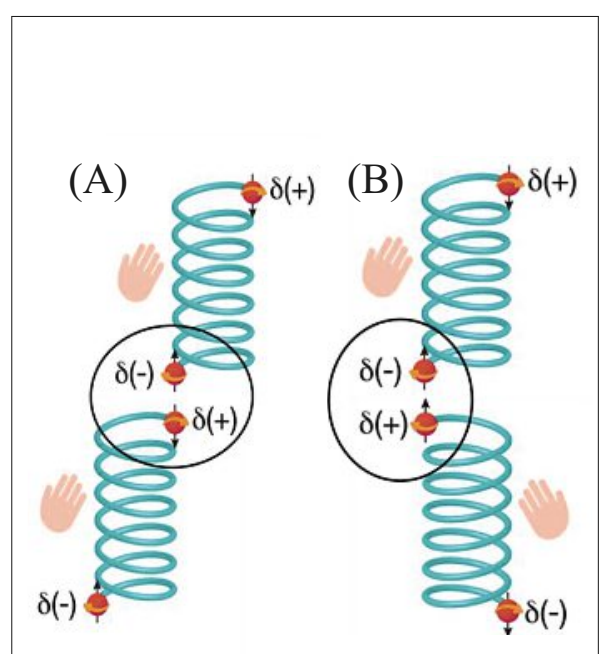

Fig. 3. The scheme illustrates spin polarization for the induced dipole-induced dipole interaction between two chiral molecules. The charge polarization (of magnitude $\delta$ and either + or - sign) is accompanied by spin (red balls with black arrows) polarization. Panel A shows a homochiral case, in which the interaction occurs for molecules that have their spin polarizations aligned opposite to each other ('singlet' potential). Panel B shows a heterochiral case, in which the interaction occurs for molecules that have their spin polarizations aligned parallel to each other ('triplet' potential).

shell, chiral molecules at close distances. In what follows we show several examples of this basic concept.

The schematic diagram in Fig. 3 illustrates how spin considerations affect the interaction between two chiral molecules. The chirality is represented by the helices, which are an example of chiral secondary structure, here; however, many molecules (including amino acids and sugars) are chiral because of the existence of asymmetric carbon atoms. As CISS relies on the helic- ity of the electron cloud, the considerations described here apply to molecules that do not possess a helical secondary structure, as well as ones that do. Moreover, the relative orientation of the two interacting molecules impacts the magnitude of the effect but does not change its essential nature, as chirality (handedness) does not depend on the direction from which the molecule is viewed. Fig. 3A shows the case of two chiral molecules (left-handed helices) of the same handedness interacting. The charge polarization of these helices is accompanied by a spin polarization that acts in the same direction; e.g. pointing outward along the helical axis (represented by the black arrow in the red circle). The region of excess charge density is shown by the circle, and the spin polarization directions of the two molecules in this region are anti-parallel (like a singlet state). Fig. $3 \mathrm{~B}$ shows the interaction of two different enantiomers (a right-handed helix and a left-handed helix), and in this case the spin polarizations are aligned parallel to each other (like a triplet state). The electronic exchange energies in these two cases are different; hence the interaction potential is enantiospecific without any consideration of the three-dimensional sterics of the interaction. Electronic structure calculations on model systems, described in ref. [12], support the model described here.

\section{Spin Polarization Accompanies Charge Polarization in Chiral Molecules}

Fig. 4A shows a schematic diagram of a new type of Hall device that we used to measure the spin polarization that accompanies the charge polarization in chiral molecules; see ref. [12]. Fig. 4B illustrates the idea of the Hall measurement. In our implementation, a constant current flows between the source (S) and drain (D) electrodes while the voltage is measured between the electrodes $\mathrm{H} 1$ and $\mathrm{H} 2$; if a magnetic field acts on the electron current then a voltage is generated between $\mathrm{H} 1$ and $\mathrm{H} 2$, perpendicular to the current flow. This Hall device is embedded under a few nanometer thick layer of $\mathrm{GaN}$ upon which a selfassembled monolayer of the molecules is adsorbed. This entire assembly is used as a working electrode that was placed into an electrochemical cell comprised of an inert electrolyte solution and a counter-electrode $(\mathrm{G})$ which is insulated from the solution. Application of a voltage between the two electrodes charges a double layer and induces a charge polarization in the chiral molecules of the film. Because the charge polarization is accompanied by a spin polarization, a voltage is generated between the two electrodes, $\mathrm{H} 1$ and $\mathrm{H} 2$. As reported in ref. [12], the electric field induced spin polarization was studied for two different lengths of an L-oligopeptide alpha-helix and compared to an achiral molecule as a control. The oligopeptides showed a Hall voltage that changed systematically with the applied bias voltage and increased as the length of the oligopeptide increased.

Fig. 5 shows the Hall voltage as a function of the gate potential for the two enantiomers of the oligopeptides: L- and D-S $\mathrm{HCH}_{2} \mathrm{CH}_{2} \mathrm{CO}-\{\mathrm{Ala}-\mathrm{Aib}\}_{5}-\mathrm{COOH}$ [Aib=2-aminoisobutyric acid or 2-methylalanine]. The $\mathrm{L}$ and $\mathrm{D}$ refer to the different handedness of the amino acid units comprising the oligopeptide. The Hall voltage observed with the chiral molecules shows a roughly linear dependence on the applied gate voltage and its sign depends on the
(A)

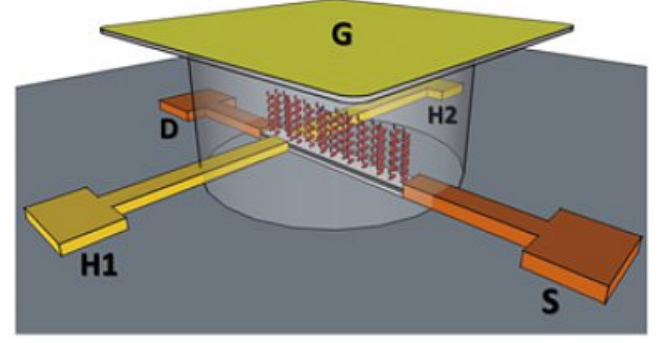

(B)

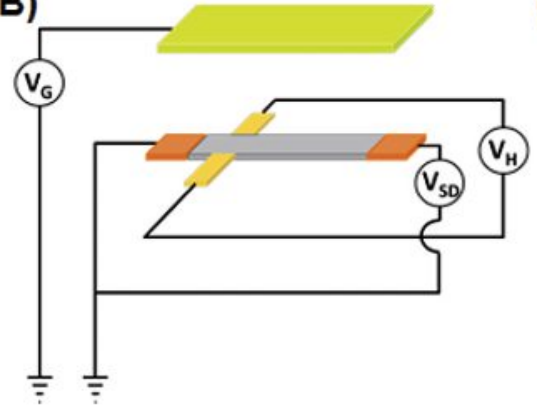

(C)

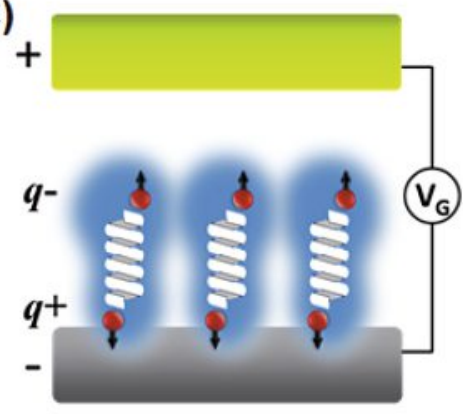

Fig. 4. Panel A shows the experimental setup in which a Hall device, coated with a self-assembled organic monolayer, is placed in solution with a counter (or gate) electrode and an inert electrolyte. When an electric potential $V_{G}$ is applied between the gate electrode (G) and the Hall device, the ionic solution is polarized so that an electric field acts on the adsorbed molecules. As a result, the molecules are polarized, due to charge reorganization (partial charges $\boldsymbol{q}+$ and $\boldsymbol{q}$-), and this induces a charge displacement in the surface region of the device. Because the charge polarization is accompanied by spin polarization (red balls with black arrows in (C)), a magnetic field that acts on the electrons flowing between the source (S) and drain (D) electrodes is also created. The Hall potential $\mathrm{V}_{\mathrm{H}}$, which is formed because of the spin magnetization, can be measured as a function of $\mathrm{V}_{\mathrm{G}}$. Panel $\mathrm{B}$ shows a schematic diagram illustrating the electrical operation of the device where $\mathrm{I}_{\mathrm{SD}}$ indicates the applied source-drain current, $\mathrm{V}_{\mathrm{G}}$ the gate voltage, and $\mathrm{V}_{\mathrm{H}}$ the differential Hall potential across the conductive channel. Panel $\mathrm{C}$ shows a scheme that illustrates the spin polarization and its generation of a magnetization at the surface. When an electric field is applied on a chiral molecule $\left(\mathrm{V}_{\mathrm{G}}\right)$ it induces charge reorganization in the molecule, resulting in spin polarization. Copied with permission from ref. [12]. 


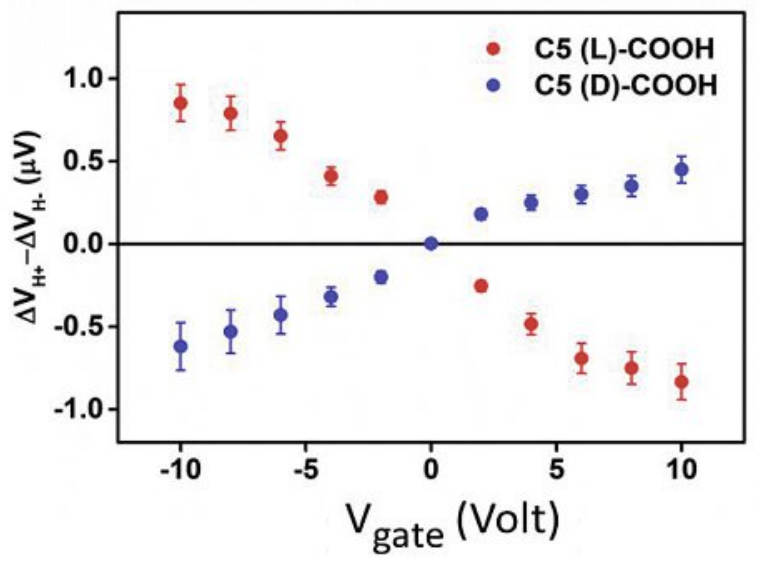

Fig. 5. Gate-dependent Hall measurements conducted on devices coated with two enantiomers of the same oligopeptides. $\mathrm{SHCH}_{2} \mathrm{CH}_{2} \mathrm{CO}$ \{Ala-Aib $\}_{5}-\mathrm{COOH}$, where Ala is alanine and Aib is $\alpha$-aminoisobutyric acid. The L- and D-enantiomers are presented in red and blue, respectively. The Hall potential was calculated as the difference between the peak response for every applied gate when the device was forward or reverse biased $\left(\Delta \mathrm{V}_{\mathrm{H}_{+}}\right.$and $\Delta \mathrm{V}_{\mathrm{H}_{-}}$, respectively). Copied with permission from ref. [12].

handedness of the molecule. The data show that charge polarization in chiral molecules results in a spin polarization. No significant signal was observed with achiral molecules; see ref. [12] for more details.

\section{Magnetization Direction Induced by Adsorption of Chiral Molecules on a Ferromagnet}

In these studies the magnetic direction of ferromagnets (FM) were shown to depend on the handedness of adsorbed chiral molecules. ${ }^{[18]}$ The substrate consisted of an epitaxial thin $\mathrm{FM}$ film, $\mathrm{Al}_{2} \mathrm{O}_{3} /$ $\mathrm{Pt} / \mathrm{Au} / \mathrm{Co} / \mathrm{Au}$. The Co FM layer is of 1.5 to $2.2 \mathrm{~nm}$ thickness and the Au coating is $5 \mathrm{~nm}$. The two enantiomers of the chiral molecules were adsorbed in selective adsorption areas on the thin film, i.e. the gold top layer. The adsorbed molecules formed self-assembled monolayers (SAM) of chiral $\alpha$-helix L- or D-polyalanine, AHPA-L and AHPA-D respectively; the AHPA oligopeptide sequence is H-CAAAAKAAA AKAAAAKAAAAKAAAAKAAAAKA AAAK-OH, where $\mathrm{C}$, A and $\mathrm{K}$ represent cysteine, lysine, and alanine respectively. These oligopeptides form an $\alpha$-helical secondary structure and are terminated with a thiol group that binds to the cobalt coated gold substrate. The force microscopy images in Fig. 6a and 6b show clearly the pattern that was used for the adsorbed SAMs.

A magnetic force microscope (MFM) was used to measure the magnetic-induced phase shift on the SAM patterns. An outof-plane magnetization, which is parallel to the oligopeptide major axis, was observed for the case of the AHPA-L regions
$10^{13}$ electrons $/ \mathrm{cm}^{2}$ is enough to reverse the magnetization direction.

Fig. 7 explains schematically how the adsorption of a chiral molecule with a specific handedness can affect the direction of the magnetic moment of a ferromagnetic (FM) substrate. Upon approaching the substrate, the chiral molecules are charge- and spin-polarized, as already explained above. The spin polarization at a specific pole is enantiospecific. If, for example, the molecule interacts with the substrate through the negative charged electric pole, then the negative pole of one of the enantiomers is mostly associated with its spin pointing towards the substrate (Fig. 7a). In this case, the Pauli repulsion between the molecule and the surface is reduced for the case in which the electrons in the FM substrate will point up. In the case that the other enantiomer approaches the surface (Fig. 7b), it will have the opposite spin alignment and therefore the interaction with the substrate will have a parallel spin alignment and larger Pauli repulsion. This 'high spin' situation is a less stable configuration; however, if the electrons in the FM can align in the opposite direction then a more stable 'low spin' configuration can be realized. The model presented here demonstrates the role of the spin-dependent exchange interaction on the binding between chiral molecules and a FM, as well as the ability of the chiral molecules to induce a change in the magnetic moment of the substrate by adsorption.

\section{Summary}

We reviewed here recent studies that demonstrate the relation between charge polarization in chiral molecules and spin polarization and the importance of this phenomenon in the interaction of chiral molecules with a ferromagnetic substrate. The observation that induced charge polarization in chiral molecules necessarily transferred between the substrate and an adsorbed molecule, the transfer of only about

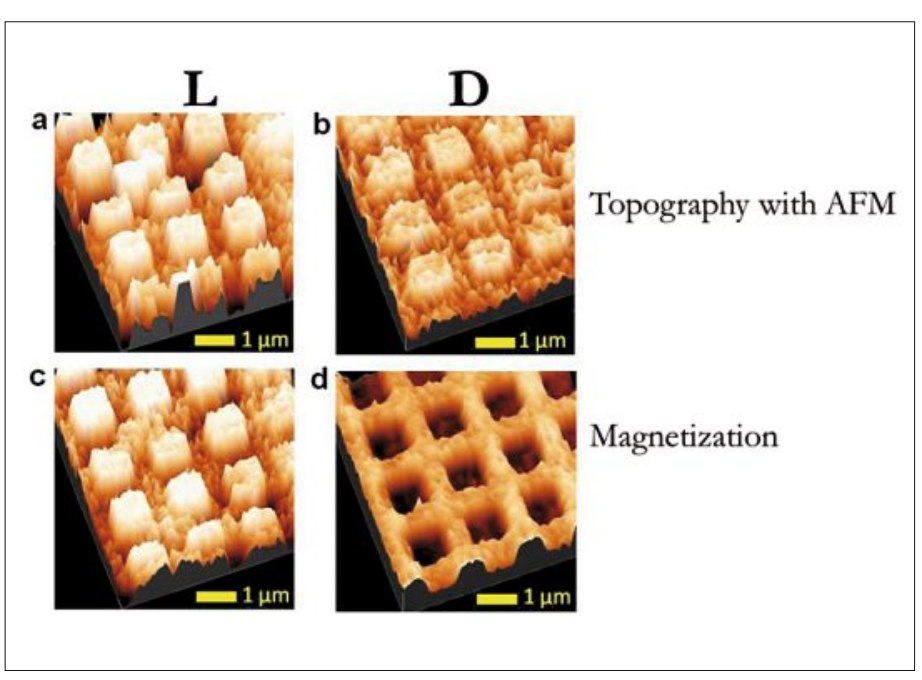

Fig. 6. Topography and magnetic phase MFM images of molecular induced magnetization orientation. Panels $a$ and $b$ show AFM topography images for the AHPA-L (a) and AHPA-D (b) films. Panels $c$ and $d$ show the corresponding MFM magnetic phase images for the same films structures; panel $c$ is AHPA-L and panel d is AHPA-D. The figure is reproduced, with permission, from ref. [18]. 
includes electron spin polarization results also in an enantiospecific term in the interaction energy between two chiral molecules. Hence it offers a new approach to enantioseparations as well as a new perspective on bio-recognition interactions. This phenomenon is likely to affect many other chiral molecules and surface interactions, such as chiral imprinting, enantiorecognition, and others.

\section{Acknowledgements}

We acknowledge the support from the John Templeton Foundation, the Israel Science Foundation, the US NSF (CHE-1464701), the Volkswagen Foundation, and the European Research Council under the European Union's Seventh Framework Program (FP7/20072013)/ERC grant agreement $n^{\circ} 338720$ CISS.

Received: April 10, 2018

[1] K. Ray, S. P. Ananthavel, D. H. Waldeck, R. Naaman, Science 1999, 283, 814.

[2] R. Naaman, D. H. Waldeck, Ann. Rev. Phys. Chem. 2015, 66, 263.

[3] K. Michaeli, N. Kantor-Uriel, R. Naaman, D. H. Waldeck, Chem. Soc. Rev. 2016, 45, 6478.

[4] a) V. R. Pasteur 'Oeuvres de Pasteur, Paris, 1922; b) A. Guijarro, M. Yus, 'The Origin of Chirality in the Molecules of Life: A Revision from Awareness to the Current Theories and Perspectives of this Unsolved Problem', RSC, 2009, Cambridge, UK.

[5] a) G. E. Uhlenbeck, S. Goudsmit, Die Naturwissenschaften 1925, 13, 953; b) G. E. Uhlenbeck, S. Goudsmit Nature 1926, 117, 264.

[6] D. E. Koshland, Angew. Chem. Int. Ed. 1994, 33, 2375.

[7] D. H. Williams, E. Stephens, D. P. O’Brien, M. Zhou, Angew. Chem. Int. Ed. 2004, 43, 6596.

[8] M. Wilchek, E. A. Bayer, O. Livnah, Immunology Lett. 2006, 103, 27.

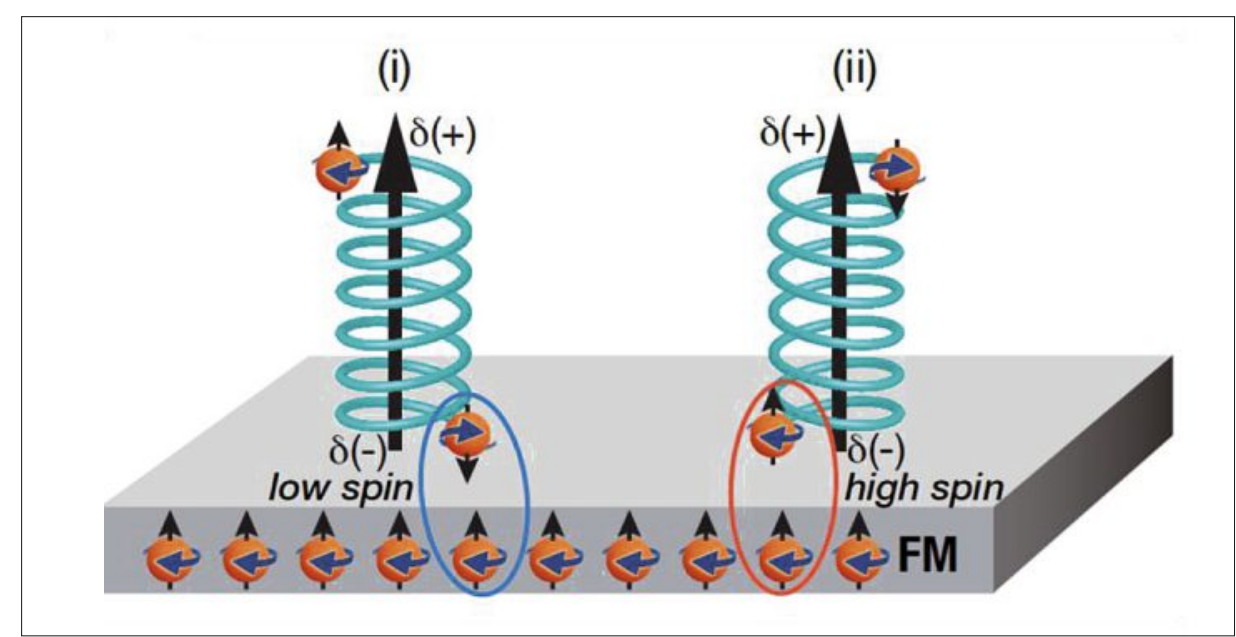

Fig. 7. A scheme of the spin-dependent interaction between chiral molecules and a ferromagnetic (FM) substrate. Panel (i) shows the case in which the spin of the unpaired electron is pointing towards the surface; and it forms a 'low spin' interface as the FM has its spins oriented up. Panel (ii) shows the case for the other enantiomer in which the spin of the unpaired electron is pointing parallel to the spins in the FM. In this case a high spin condition is formed if the FM spins are oriented up.

[9] See for example: A. S. Christensen, T. Kubar, Q. Cui, M. Elstner, Chem. Rev 2016, 116, 5301 .

[10] J. R. Wagner, C. T. Lee, J. D. Durrant, R. D. Malmstrom, V. A. Feher, R. E. Amaro, Chem. Rev. 2016, 116, 6370.

[11] K. Banerjee-Ghosh, O. Ben Dor, F. Tassinari, E. Capua, S. Yochelis, A. Capua, S.-H. Yang, S. S P. Parkin, S. Sarkar, L. Kronik, L. T. Baczewski, R. Naaman, Y. Paltiel, Science 2018, doi:1126/ science.aar4265.

[12] A. Kumar, E. Capua, M. K. Kesharwani, J. M. L. Martin, E. Sitbon, D. H. Waldeck, R. Naaman, Proc. Natl. Acad. Sci. USA 2017, 114, 2474.

[13] K. Michaeli, V. Varade, R. Naaman, D. Waldeck, J. Physics: Cond. Matt. 2017, 29, 103002.

[14] A. J. Stone 'The Theory of Intermolecular Forces', 2013, Oxford University Press, ISBN 978-0-19-967239-4.
[15] K. Szalewicz, Wiley Interdisciplinary Reviews: Computational Molecular Science 2012, 2, 187.

[16] I. Langmuir, J Am. Chem. Soc. 1919, 41, 868.

[17] M. Massimi, 'Pauli's Exclusion Principle', 2005, Cambridge University Press, ISBN $0-521-83911-4$

[18] O. Ben Dor, S. Yochelis, A. Radko, K. Vankayala, E. Capua, A. Capua, S.-H. Yang, L. T. Baczewski, S. S. P. Parkin, R. Naaman, Y. Paltiel, Nat. Comm. 2017, 8, 14567.

[19] M. Tsoi, A. G. M. Jansen, J. Bass, W.-C. Chiang, M. Seck, V. Tsoi, P. Wyd, Phys. Rev. Lett. 1998, 80, 4281 .

[20] E. B. Myers, D. C. Ralph, J. A. Katine, R. N. Louie, R. A. Buhrman, Science 1999, 285, 867. 